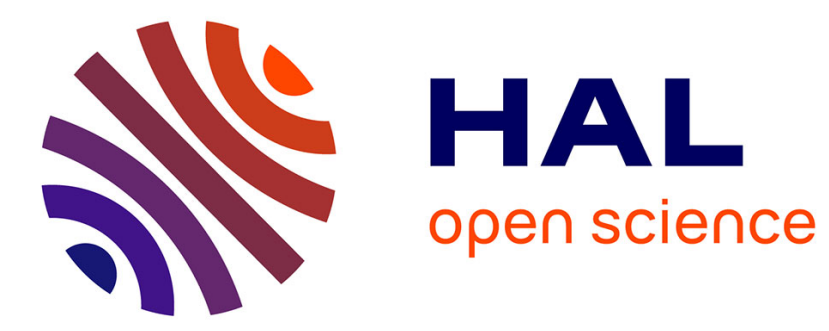

\title{
Magnetic properties of an iron-Nickel single crystal ordered by neutron bombardment
}

\author{
Louis Néel, J. Pauleve, R. Pauthenet, J. Laugier, D. Dautreppe
}

\section{To cite this version:}

Louis Néel, J. Pauleve, R. Pauthenet, J. Laugier, D. Dautreppe. Magnetic properties of an iron-Nickel single crystal ordered by neutron bombardment. Journal of Applied Physics, 1964, 35 (3), pp.873-876. hal-02887454

\section{HAL Id: hal-02887454 \\ https://hal.science/hal-02887454}

Submitted on 2 Jul 2020

HAL is a multi-disciplinary open access archive for the deposit and dissemination of scientific research documents, whether they are published or not. The documents may come from teaching and research institutions in France or abroad, or from public or private research centers.
L'archive ouverte pluridisciplinaire HAL, est destinée au dépôt et à la diffusion de documents scientifiques de niveau recherche, publiés ou non, émanant des établissements d'enseignement et de recherche français ou étrangers, des laboratoires publics ou privés. 


\title{
Magnetic Properties of an Iron-Nickel Single Crystal Ordered by Neutron Bombardment
}

\author{
L. Nfel, J. Pauleve, R. Pauthenet, J. Laugier, and D. Dautreppe \\ Centre d'Etudes Nucléaires et Laboratoire d'Electrostatique et de Physique du Métal, Grenoble, France
}

\begin{abstract}
It has been shown that a new ordered structure can be obtained in an $\mathrm{Fe}-\mathrm{Ni}(50 \%-50 \%)$ alloy, by irradiating it with neutrons below $320^{\circ} \mathrm{C}$. This ordered structure is tetragonal (AuCu type) and leads to a very high magnetic anisotropy. We have studied the magnetic properties of a single-crystal disk irradiated with neutrons at $295^{\circ} \mathrm{C}$, with an applied magnetic field along the [100] direction. The $\mathrm{x}$-ray superlattice lines show that after irradiation the sample is made of small ordered crystallites with their tetragonal axes distributed along a $[100]$, [010], or [001] direction. The study of the anisotropy energy in the sample plane [011], and the magnetization curves drawn for different directions, show that this sample presents the characteristics of a uniaxial substance. But if we express, in a classical way, the anisotropy energy by $K_{1} \sin ^{2} \theta+K_{2} \sin ^{4} \theta$, we find experimentally $K_{1}=3.2 \cdot 10^{6} \mathrm{ergs} / \mathrm{cm}^{3}$ and $K_{2}=2.3 \cdot 10^{6} \mathrm{ergs} / \mathrm{cm}^{3}$.

This high value of $K_{2}$ is not in agreement with the conception of independent crystallites. One of us (L.N.) has made a theory taking into account the exchange interaction between the crystallites which are in fact smaller than the magnetic domains. The formulas obtained in this way give a good agreement with the experimental results.
\end{abstract}

\section{EXPERIMENTAL RESULTS}

$\mathbf{T}$ IHE existence of an order-disorder transition in an iron-nickel alloy $(50 \%-50 \%)$ has been established by bombarding a polycrystalline sample with neutrons. ${ }^{1}$ The critical temperature of the ordered state is $320^{\circ} \mathrm{C}$, and this ordering induces a very large magnetic anisotropy. In order to ascertain the physical properties of this transition we have irradiated in the swimming pool reactor "Melusine", a single crystal of $\mathrm{Fe}-\mathrm{Ni}$ (50-50, on atomic basis) in the form of a disk, $9.3 \mathrm{~mm}$ in diameter and $1.1 \mathrm{~mm}$ thick, cut from a (011) plane. The sample, heated to $295^{\circ} \mathrm{C}$ while subject to a uniform magnetic field of 2500 Oe along the [100] direction of the crystal, was irradiated by $1.5 \times 10^{20}$ neutrons $/ \mathrm{cm}^{2}$ of which $2.1 \times 10^{19}$ had more than $1-\mathrm{MeV}$ energy. The $\mathrm{x}$-ray study of the sample after bombardment confirms the establishment of an ordered structure of the type $\mathrm{AuCu}$, without any appreciable quadratic distortion (less than $5 \times 10^{-4}$ ); it also indicates that the sample is composed of quadratic crystallites and that all the quaternary axes are along one of the three directions $[100]$, [010], and [001] of the single crystal. If one designates by $p$ and $S_{p}$ the proportion by volume and the long range order parameter of the crystallites having their quadratic axis along the $[100]$ direction, by $q$ and $S_{q}, r$ and $S_{r}$ the same meanings relative to the crystallites having their quadratic axis along the [010] and [001] directions, then a study of the x-ray superstructure lines allows one to find: $S_{p} p^{\frac{1}{2}}=$ $0.32 \pm 0.01 ; S_{q} q^{\frac{1}{2}}=S_{r} r^{\frac{1}{2}}=0.21 \pm 0.05 ;$ and to determine the mean size $L$ of the ordered crystallites to be about $300 \AA$.

We have measured at $293^{\circ} \mathrm{K}$ and in a field $H_{e}$ up to $30 \mathrm{kOe}$, the components of the magnetization $J_{\| 1}$ and $J_{\perp}$, that are, respectively, parallel and perpendicular to the field for the directions [100], [001], and [111].

${ }^{1}$ J. Pauleve, D. Dautreppe, J. Laugier, and L. Néel, J. Phys. Radium 23, 841 (1962).
When the field $H_{e}$ is directed in a [100] direction, $J_{\perp}$ is zero and $J_{\| 1}$ starts to rise linearly with the field (see curve a, Fig. 1) up to about 1600 Oe with a measured susceptibility equal to the inverse of the disk's demagnetization coefficient. For larger fields saturation is reached; the intensity of the magnetization at saturation is equal to $1300 \mathrm{emu}$; it differs only slightly from that measured on a nonirradiated sample. For a field along the [011] direction, the substance is very hard to magnetize (curve b, Fig. 1); within the accuracy of our experiments $J_{\perp}$ is zero and $J_{11}$ increases up to a field of about $17 \mathrm{kOe}$. The anisotropy energy required to reorient the magnetization from the $[100]$ to the [011] direction is equal to $6.7 \times 10^{6} \mathrm{ergs} / \mathrm{cm}^{3}$. For $H_{e}$ parallel to [111] (curves $c$ and d, Fig. 1), $J_{\|}$ and $J_{\perp}$ start to increase linearly with the field up to a value of about $2500 \mathrm{Oe}$; for larger fields $J_{11}$ continues to rise and approaches saturation with the classical law, while $J_{\perp}$ decreases and goes to zero. These results show a great similarity to those observed on a single crystal of hexagonal cobalt. ${ }^{2}$ They show us that the ordered crystal of $\mathrm{Fe}-\mathrm{Ni}$ has the characteristics of a uniaxial substance with the easy direction of magnetization along the [100] axis and the hard direction of magnetization along the [011] axis and that it is magnetized by a mechanism of phases of magnetic domains. ${ }^{3}$ If, as in the case of a uniaxial homogeneous substance, one represents the anisotropy energy by $K_{1} \sin ^{2} \theta+K_{2} \sin ^{4} \theta$, where $\theta$ is the angle between the magnetization and the $[100]$ direction, the analysis ${ }^{4}$ of the curve $\left(J_{11}, H_{e}\right)$ along the [011] direction shows that the constants $K_{1}$ and $K_{2}$ are of the same order of magnitude and, respectively, equal to $3.2 \cdot 10^{6}$ and $2.3 \cdot 10^{6} \mathrm{ergs} / \mathrm{cm}^{3}$.

\footnotetext{
2 Y. Barnier, R. Pauthenet, and G. Rimet, Compt. Rend. 252 , 3024 (1961).

${ }^{3}$ L. Néel, J. Phys. Radium 12, 431 (1951).

${ }^{4}$ R. Pauthenet, Y. Barnier, and G. Rimet, J. Phys. Soc. Japan 17,309 (1962)
} 


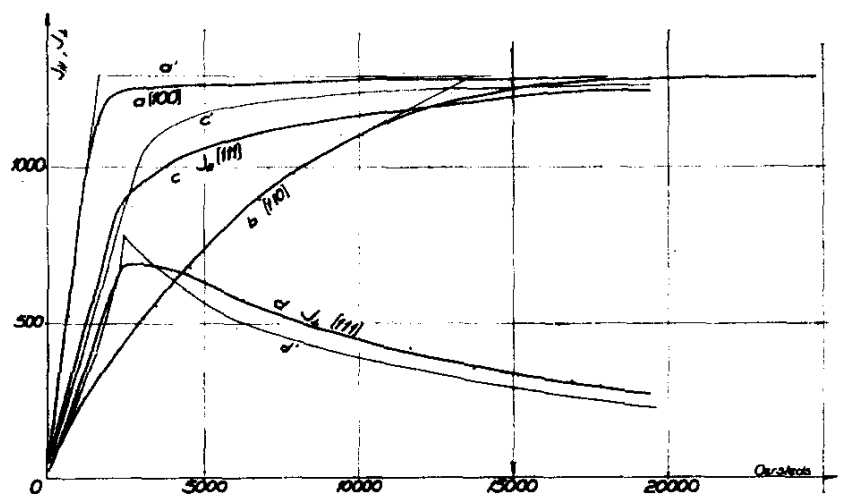

Fig. 1. Variation of magnetization components vs magnetic field for different crystallographic directions of an ordered $\mathrm{Fe}-\mathrm{Ni}$ single crystal.

\section{THEORY OF THE MAGNETOCRYSTALLINE ENERGY OF A MACROCRYSTAL SUBDIVIDED INTO QUADRATIC CRYSTALLITES}

It is difficult to understand a priori the large value of $K_{2}$ as well as the fact that it is of the same order of magnitude as $K_{1}$; on the other hand, experiments show that a weak magnetic field saturates the sample in the direction of the field applied during treatment. One may, therefore, conclude that the crystallites cannot be considered as independent of one another since several among them possess a direction of hard magnetization which coincides with the direction of easy magnetization of the macrocrystal. One can imagine that the dimensions of the crystallites being probably smaller than the dimensions of the elementary domains, there exists a close correlation between the direction of spontaneous magnetization $J_{8}$ for the different crystallites. Being given a certain direction of the macroscopic magnetization $J$, defined by the direction cosines $\alpha, \beta, \gamma$, with respect to the tetragonal axes of the macrocrystal, the deviations of the local spontaneous magnetization with respect to this direction at the different points in the different crystallites will always be small through the equalizing action of the exchange forces and the internal magnetic fields which cause scattering.

Let us consider, therefore, a macrocrystal made of three types of quadratic crystals 1,2 , and 3 , whose tetragonal axis is along one or the other of the three tetragonal axes of the crystal and which respectively occupy the relative volumes $p ; q, r$, with $p+q+r=1$. The simplest expressions that one can choose in order to represent the magnetocrystalline energy density are, for the three types, respectively, $-P \alpha_{0}{ }^{2},-Q \beta_{0}{ }^{2},-R \gamma_{0}{ }^{2}$; where $\alpha_{0}, \beta_{0}$, and $\gamma_{0}$, are the direction cosines of the spontaneous magnetization. Each crystallite is approximately a cube of length $L$ and is surrounded by other crystallites which are randomly distributed amongst the three types; the total number, of the order of a dozen, is sufficiently large so that the actual surroundings may be replaced by homogeneous surroundings with a uniform magnetization $J$ approximately equal to the spontaneous magnetization $J_{s}$, in the direction $\alpha, \beta, \gamma$, coinciding with the direction of the macroscopic magnetization.

The result is that all the crystallites of the same type, when under identical external conditions, possess the same magnetization defined at the center of the crystallite by the direction cosines $\alpha+\lambda_{i}, \beta+\mu_{i}, \gamma+\nu_{i}$. This magnetization must join continuously at the boundary of the crystallite with the magnetization, of direction $\alpha, \beta, \gamma$, of the fictitious surroundings. To express this condition we write:

$$
F=\sin (\pi x / L) \sin (\pi y / L) \sin (\pi z / L),
$$

where a corner of the cubic crystallite is the origin of the coordinates system and $F$ is essentially positive. It suffices then to assume that at the different points of each crystallite the direction cosines that define the local direction of the spontaneous magnetization are respectively equal to

$$
\alpha+\lambda_{i} F, \quad \beta+\mu_{i} F, \quad \gamma+\nu_{i} F \quad(i=1,2,3) .
$$

The $\lambda_{i}, \mu_{i}, \nu_{i}$, which are assumed to be small compared to one, obey the three relations

$$
\alpha \lambda_{i}+\beta \mu_{i}+\gamma \nu_{i}=0
$$

meaning that by neglecting the second-order terms, the sum of the squares of the direction cosines is equal to one. The three relations of the type

$$
p \lambda_{1}+q \lambda_{2}+r \lambda_{3}=0
$$

with the two other relations in $\mu_{i}$ and $\nu_{i}$, connote that the direction of the mean magnetization is truly the direction $\alpha, \beta, \gamma$.

After having thus defined the direction of the magnetization at all the points of the macrocrystal we calculate the different types of corresponding energies.

The magnetocrystalline energy density $E_{c}$. This density is the mean density relative to the different points of the crystallite; which gives, if one takes into account that the mean value of $F$ is equal to $8 / \pi^{3}$ :

$$
\begin{aligned}
& -E_{c_{1}}=P\left[\alpha^{2}+\left(16 / \pi^{3}\right) \alpha \lambda_{1}\right] ; \\
& -E_{c_{2}}=Q\left[\beta^{2}+\left(16 / \pi^{3}\right) \beta \mu_{2}\right] ; \\
& -E_{c_{3}}=R\left[\gamma^{2}+\left(16 / \pi^{3}\right) \gamma \nu_{3}\right] .
\end{aligned}
$$

We have neglected the terms $\lambda_{1}{ }^{2}, \mu_{2}{ }^{2}, \nu_{3}{ }^{2}$ which are second order.

The magnetostatic energy density in the external field $E_{m}$. Noticing that the angle $\phi$ of the local magnetization with the mean magnetization is given by $\phi^{2}=\left(\lambda_{i}{ }^{2}+\mu_{i}{ }^{2}+\nu_{i}{ }^{2}\right) F^{2}$ and that the average value of $F^{2}$ is equal to $\frac{1}{8}$; the sought-for density is written as:

$$
E_{m_{i}}=-H_{T} J_{s}\left[1-\frac{1}{16}\left(\lambda_{i}^{2}+\mu_{i}^{2}+\nu_{i}^{2}\right)\right] \text {, }
$$

where $H_{T}=\alpha H_{x}+\beta H_{y}+\gamma H_{z}$; that is to say, the projection of the external field $H$ on $J_{s}$.

The exchange energy density $E_{w}$. This term comes 
from the increase of the exchange energy which is related to the nonuniformity of the magnetization and which is calculated by the Landau-Lifshitz formula:

$E_{v}=\frac{1}{12} N a_{0}^{2}\left[\left(\operatorname{grad} J_{x}^{2}\right)+\left(\operatorname{grad} J_{y}\right)^{2}+\left(\operatorname{grad} J_{z}\right)^{2}\right]$,

where $N$ is the molecular field coefficient, $a_{0}$ is the distance between the nearest-neighbor atoms in the crystalline lattice, and $J_{x}, J_{y}, J_{z}$ are the projections of $J_{s}$ on the three coordinate axes. The sought-for density is written as:

$$
E_{w_{i}}=\left(\pi^{2} / 32\right) N J_{s}{ }^{2}\left(a_{0}{ }^{2} / L^{2}\right)\left(\lambda_{i}{ }^{2}+\mu_{i}{ }^{2}+\nu_{i}{ }^{2}\right) .
$$

The energy density of the internal scattering fields $E_{d}$. Each crystallite is submerged in a uniform magnetic field which is nearly equal to $\frac{4}{3} \pi J_{s}$ and at each point the local magnetization of the crystallite makes with $J_{s}$ the angle $\phi$ calculated above. The corresponding increase of energy is given by

$$
E_{d_{i}}=\frac{4}{3} \pi J_{s}{ }^{2}\left(\left\langle\phi^{2}\right\rangle_{\mathrm{av}} / 2\right)=\left(\pi J_{\mathrm{s}}{ }^{2} / 12\right)\left(\lambda_{i}{ }^{2}+\mu_{i}{ }^{2}+\nu_{i}{ }^{2}\right) .
$$

We must not take into account the nonuniformity of the magnetization inside each crystallite: each crystallite is comparable, in fact, to a series of concentric spherical shells. Each shell possesses a uniform magnetization and so the internal field is zero and there is no magnetostatic interaction between two shells.

The mean total energy density $W_{i}$ of a crystallite is thus equal to $E_{c_{i}}+E_{m_{i}}+E_{w_{i}}+E_{d_{i}}$; the total energy density $W_{M}$ of the macrocrystal is equal to $p W_{1}+$ $q W_{2}+r W_{3}$ and is written

$$
\begin{aligned}
& W_{M}=-(\left.p P \alpha^{2}+q Q \beta^{2}+r R \gamma^{2}\right)-H_{T} J_{s} \\
&-\left(16 / \pi^{3}\right)\left(p P \alpha \lambda_{1}+q Q \beta \mu_{2}+r R \gamma \nu_{3}\right) \\
&+p C\left(\lambda_{1}{ }^{2}+\mu_{1}{ }^{2}+\nu_{1}^{2}\right)+q C\left(\lambda_{2}{ }^{2}+\mu_{2}{ }^{2}+\nu_{2}{ }^{2}\right) \\
&+r C\left(\lambda_{3}{ }^{2}+\mu_{3}{ }^{2}+\nu_{3}{ }^{2}\right),
\end{aligned}
$$

where

$$
C=\left(H_{T} J_{s} / 16\right)+\left(\pi^{2} N J_{s}{ }^{2} a_{0}^{2} / 32 L^{2}\right)+\left(\pi J_{s}^{2} / 12\right) .
$$

Considering the $\alpha, \beta, \gamma$ as constants, we determine the nine unknowns $\lambda_{i}, \mu_{i}, \nu_{i}$, which appear to verify the six Eqs. (2) and (3), in such a manner that the energy $W_{M}$ is a minimum. The rather long calculation is performed without any difficulty by utilizing the method of Lagrangian multipliers - one obtains finally

$$
W_{c}=-\left(\alpha^{2} p P+\beta^{2} q Q+\gamma^{2} r R\right)-\left(64 / \pi^{6}\right)(D / C),
$$

where

$$
\begin{aligned}
D=\alpha^{2}\left(1-\alpha^{2}\right) p(1-p) & P^{2}+\beta^{2}\left(1-\beta^{2}\right) q(1-q) Q^{2} \\
+\gamma^{2}\left(1-\gamma^{2}\right) & r(1-r) R^{2}+2 \beta^{2} \gamma^{2} q r Q R \\
& +2 \gamma^{2} \alpha^{2} r p R P+2 \alpha^{2} \beta^{2} p q P Q .
\end{aligned}
$$

We thus obtain the expression $W_{c}$ of the energy density for the macrocrystal as a function of the direction cosines of the average magnetization; that is to say, the magnetization which is macroscopically observable. Everything happens as if we observed a homogeneous sample, with a uniform magnetization equal to $J$ and endowed with an apparent magnetocrystalline energy $W_{c}$. The reason we have not included in the expression for $W_{c}$, the term $-H_{T} J_{s}$, which was in $W_{M}$, is that it represents the energy of the magnetized macrocrystal in an external field, and it is necessary to separate it from $W_{c}$. We notice that the energy $W_{c}$ is a function of the 4th degree in the direction cosines, though the magnetocrystalline energy of the constituent crystallites only contains second-order terms; thus the difficulty relative to the term $K_{2}$ is removed. Nevertheless there exists a noticeable difference between this case and that of a homogeneous substance. It is that the apparent spontaneous magnetization $J$ is not constant, but is related to the true spontaneous magnetization $J_{s}$ by the relation

$$
J=J_{s}\left[1-\left(\left\langle\phi^{2}\right\rangle_{\mathrm{av}} / 2\right) .\right.
$$

One easily finds that

$$
\left\langle\phi^{2}\right\rangle_{\mathrm{av}}=\left(8 / \pi^{6}\right)\left(D / C^{2}\right)
$$

and that $J$ is a function of $H_{T}$ and of $\alpha, \beta, \gamma$.

When the neutron bombardment is performed in the absence of a magnetic field or when the field is parallel to a ternary axis of the macrocrystal, $W_{c}$ is greatly simplified and becomes

$$
W_{c}^{\prime}=\left(64 / 3 \pi^{6}\right)\left(p^{2} / c\right)\left(\alpha^{4}+\beta^{4}+\gamma^{4}\right) .
$$

The expression for the apparent magnetocrystalline energy only contains terms of the 4th degree in $\alpha, \beta, \gamma$.

In the case studied experimentally, that is, of irradiation performed with a magnetic field parallel to the [100] axis of the macrocrystal, one must have, by symmetry, $q=r$ and $Q=R$. As a function of the angle $\theta$ that the mean macroscopic magnetization $J$ makes with the $[100]$ axis, $W_{c}$ is written as

$$
W_{c}=\sin ^{2} \theta(p P-q Q)-\left(64 / \pi^{6}\right)(D / C)
$$

with

$$
\begin{array}{r}
D=\sin ^{2} \theta \cos ^{2} \theta\left[p(1-p) P^{2}+q(1-q) Q^{2}+2 p q P Q\right] \\
+\left(\sin ^{4} \theta / 2\right) q Q^{2} .
\end{array}
$$

\section{INTERPRETATION OF EXPERIMENTAL RESULTS}

In writing the equations of equilibrium for the magnetization in the external field for four different values of the field, we can determine, by successive approximations, the values

$$
\begin{gathered}
L / a_{0}=50 ; \quad p=0.6 ; \quad q=0.2 ; \\
P=13.7 \times 10^{6} \mathrm{ergs} / \mathrm{cm}^{3} ; \quad Q=10.3 \times 10^{6} \mathrm{ergs} / \mathrm{cm}^{3}
\end{gathered}
$$

which is in satisfactory agreement with the experimental curves $\left(J, H_{e}\right)$ (Fig. 1). 


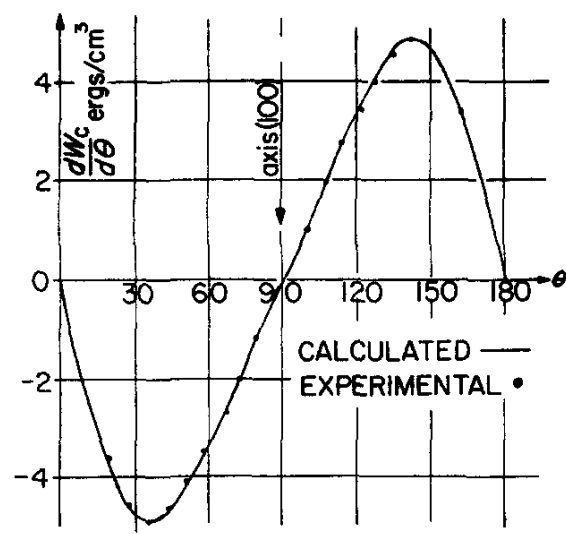

Fig. 2. Torque $d W / d \theta$ due to magnetic anisotropy vs $\theta$, angle between magnetization and [011] axis.

The study of the magnetic anisotropy with the torque magnetometer, in a field of 10000 Oe gives similar results. The experimental variation of the torque as a function of $\theta$ may be represented in a satisfactory manner by the curve $\left(d W_{c} / d \theta, \theta\right)$ (Fig. 2) when using the values

$$
\begin{gathered}
L / a_{0}=50 ; \quad p=0.6 ; \quad q=0.2 ; \\
P=11.6 \times 10^{6} \mathrm{ergs} / \mathrm{cm}^{3} ; \quad Q=10 \times 10^{6} \mathrm{ergs} / \mathrm{cm}^{3} .
\end{gathered}
$$

Taking into account the approximations made in the theory, of the fact that the demagnetization field inside the disk is not uniform, and of the experimental difficulties encountered, the agreement between the two sets of values is gratifying.

In the absence of an external field, the substance is not magnetized; as for a homogeneous material, one can imagine that the crystal is formed by two phases of magnetic domains, in equal proportions, of which the mean magnetizations $J$ are antiparallel and along the $[100]$ axis. When a small external field is applied along the [111] axis, it is necessary that the internal field $H_{i}$ is directed along [011] so that the two types of domains are in equilibrium. The mean magnetizations in the two types of domains make the same angle with the [100] direction, and the proportion $u$ of domains with their magnetization along the direction of $H_{e}$ with respect to [011] increases while the proportion $v$ of the other type decreases. The variation of the magnetization is mainly due to the displacements of the domain walls. This mechanism exists for field up to about 2500 Oe where $u$ assumes the value 1 . For stronger fields, the variation of the magnetization is due to the rotation of the mean magnetization. We have thus calculated the variations $\left(J_{16}, H_{e}\right)$ and

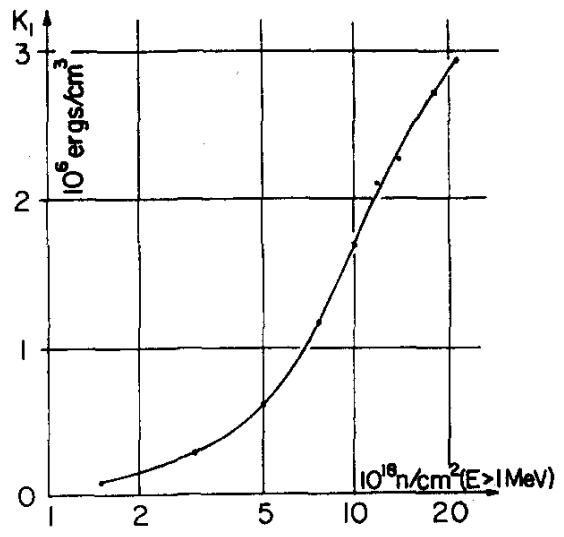

FIG. 3. $K_{1}$ variation vs dose of neutrons during irradiation of an $\mathrm{Fe}-\mathrm{Ni}$ single crystal with an applied magnetic field along the $[100]$ axis.

$\left(J_{\perp}, H_{e}\right)$ (curves $\mathrm{c}^{\prime}$ and $\mathrm{d}^{\prime}$, Fig. 1) by taking for the anisotropy energy the expression $W_{c}$. With the first group of values for the parameters, the agreement is satisfactory.

\section{CONCLUSION}

From these results we note that if $a_{0}$ is about $3 \AA$, $L$ is equal to $150 \AA$; that is to say, of an order of magnitude which is corroborated by $x$-ray analysis, the angle between the spontaneous and mean magnetizations is always small; the calculations show that the term $\left\langle\phi^{2}\right\rangle_{\text {av }} / 2$ is generally smaller than 0.04 . The ratio $P / Q$ is about 1.2 , so that the ratio $p / q$ is equal to 3 . One may conclude that during irradiation the applied field has only a slight influence on the values of the anisotropy, that the magnetic energies must remain small compared to the binding energies between nearest neighbors that are responsible for the order, while on the other hand the field favors the development of the ordering in the direction in which it is applied. The calculated values are, respectively (order of magnitude), $S_{p}=0.41$ and $S_{q}=0.45$.

In conclusion, the ensemble of the properties of the $\mathrm{Fe}-\mathrm{Ni}$ alloy ordered by neutron bombardment while in a magnetic field, are explained by the theory developed by one of the authors (L. N.). To verify these results we have begun some studies on spherical single crystals in order to reduce the effect of the inhomogeneity from the demagnetization field. We also propose to study the kinetics of the transformation since, as can be shown from the variation of $K_{1}$ as a function of the neutron flux (Fig. 3), the transformation is far from completion. 